\title{
Propiedades estructurales, electrónicas y magnéticas de la perovskita $\mathrm{BiRO}_{3}$
}

\author{
C. E. Deluque Toro 2,4 \\ D. A. Landínez Téllez ${ }^{1}$ Jairo Arbey Rodríguez M. ${ }^{3} \quad$ J. Roa-Rojas ${ }^{1}$ \\ A. Mosquera $^{2}$
}

Recibido:

Abril 12 de 2011

Aceptado:

Diciembre 10 de 2011

\section{Resumen}

Presentamos un estudio detallado de las propiedades estructurales, los estados, electrónicos y magnéticos de las perovskitas del tipo $\mathrm{BiRO}_{3}$, con $R=\mathrm{Fe}, \mathrm{Co}$, Ni en su estructura cúbica, con grupo de simetría Pm-3m. En la obtención de los resultados se utilizó el método de Spin Polarizado de Ondas Planas Aumentadas y Linealizadas (SP-FP-LAPW) dentro del marco de la teoría DFT. El estudio estructural consistió en calcular las energías de cohesión en función del volumen y mediante su ajuste a la ecuación de estado de Murnaghan se determinaron el módulo de volumen, el volumen de equilibrio y de éste el valor de constante de red. En el estudio de las características electrónicas se han determinado curvas de energía en contra del número de onda ( $k$ ) y la densidad de estado (DOS) para cada polarización de spin.

Palabras clave: perovskitas $\mathrm{BiRO}_{3}$, DFT, propiedades electrónicas.

\section{Abstract}

We present a detailed study about the structural properties, electronic and magnetic states $\mathrm{BiRO} 3$ perovskites, with $\mathrm{R}=\mathrm{Fe}, \mathrm{Co}, \mathrm{Ni}$, cubic structure and group of symmetry Pm-3m. By obtaining results was used the Spin polarized Full-Potential Linear Augmented Plane Wave method (SP-FP-LAPW) into the framework the Theory Functional Density (DFT). The structural study consist to calculate the cohesion energy as a function of volume and by means of the Murnaghan state equation, bulk modulus, equilibrium volume and lattice constant was determinated. Studying the electronic characteristic the energy curves in function of wave number $(k)$ and the density of states (DOS) with polarized spin was calculated.

Keywords: Perovskites BiRO3, DFT, Electronic Properties.

\section{Introducción}

3. GEMA, Grupo de Estudio de Materiales, Departamento de Física, Universidad Nacional de Colombia, Bogotá DC

4. Departamento de Física, Universidad Nacional del Comahue, Neuquén, Argentina.

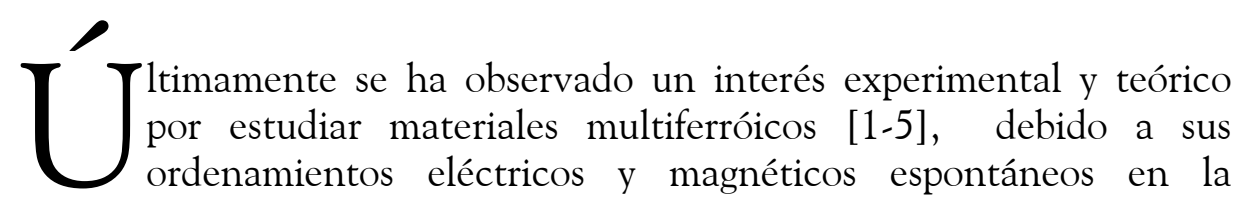


misma fase [6], esto es, coexistencia de ferromagnetismo y ferroeléctricidad y sus posibles aplicaciones tecnológicas. A pesar de que los mecanismos que favorecen tanto la existencia como la interacción entre los ordenamientos magnéticos y eléctricos no han sido comprendidos aún se tiene un gran interés en el estudio de estos materiales ya que la introducción de un grado de libertad adicional permitiría la creación de dispositivos que pueden ser controlados tanto por campos eléctricos como campos magnéticos.

El $\mathrm{BiRO}_{3}$ es un óxido con metales de transición, estudios realizados reportan que el compuesto $\mathrm{BiRO}_{3}$ presenta cambio de estructura al aumentar la presión o la temperatura, la perovskita $\mathrm{BiFeO}_{3}[7,8]$ pasa de una estructura romboédrica a tetragonal

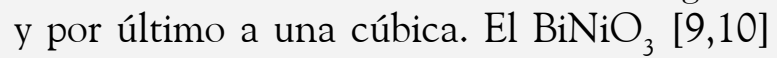
puede pasar de una estructura tetragonal a una triclínica después pasa a cúbica y el compuesto $\mathrm{BiCoO}_{3}[11,12]$ sufre una transformación de tetragonal a ortorrómbica y por último pasa a una estructura cúbica. Experimentalmente, se observa que para temperaturas $\mathrm{O}$ presiones muy altas el compuesto $\mathrm{BiRO}_{3}$ presenta la fase cúbica $(\mathrm{Pm}-3 \mathrm{~m})$. Por ejemplo, para el compuesto $\mathrm{BiFeO}_{3}$ usando la técnica de DRX, S. A. T. Redfern et al., [8] confirman la existencia de la transición de la fase ortorrómbica a cúbica (Pm-3m) a temperatura de $930^{\circ} \mathrm{C}$ o también a presiones de $\sim 45 \mathrm{Gpa}$, pero estos autores no realizaron refinamiento de los parámetros a dichos resultados. Posteriormente Belik et al. [7] mediante la técnica de alta resolución de difracción de polvo con rayos X de sincrotrón a la temperatura ambiente y hasta presiones de 9,7 Gpa, Observó que hay transformaciones, desde una fase romboédrica hacia una fase monoclínica, la cual después se transforma en ortorrómbica. Y mediante el refinamiento obtuvieron la información estructural en las diferentes fases.

Para el material $\mathrm{BiNiO}_{3}$ experimentalmente Y. Ya. Tomashpoískii et al., [9] reportan la estructura cúbica $(\mathrm{Pm}-3 \mathrm{~m})$ como fase que aparece a altas presiones. En un trabajo más reciente Masaki Takata [10] reporta la existencia de una nueva fase triclínica para presiones de $6 \mathrm{Gpa}$. Esta nueva estructura fue encontrada mediante la técnica de difracción de sincrotrón de rayos $\mathrm{X}$ en polvo.

En cuanto el $\mathrm{BiCoO}_{3}$, Alexei A. Belik et al., [11] confirman la existencia de la estructura tetragonal a partir de difracción de neutrones a temperaturas desde 5 hasta 520 K. En un trabajo más reciente [12] y mediante combinación de técnica de sincrotrón de rayos $\mathrm{X}$ y difracción de neutrones reportan los cambios de fase que sufre el sistema al aumentar la presión. Los autores observan que para una presión de 0.1 MPa su estructura es tetragonal y después, para una presión de 5.8 GPa observan una estructura ortorrómbica; por último a presiones muy altas observan la fase cúbica $(\mathrm{Pm}-3 \mathrm{~m})$. Por estos resultados experimentales sabemos que la fase cúbica existe.

Sin embargo, a pesar que hay mucha literatura basada en primeros principios referente a la estructura romboédrica, tetragonal, ortorrómbica, triclínica y monoclínica [11,13-17], la fase cúbica de la perovskita $\mathrm{BiRO}_{3}$ no ha sido estudiada teóricamente, aunque, experimentalmente ha sido reportada su existencia por diferentes investigadores. Esta fase se presenta para alta temperatura y alta presión. En el presente trabajo presentamos un estudio detallado de las propiedades estructurales, electrónicas y magnéticas del compuesto $\mathrm{BiRO}_{3}$ ya que es una fase experimental.

\section{Método de cálculo}

Los cálculos se realizaron con spin polarizado dentro del marco de la teoría de funcional de densidad (DFT) [18], mediante el código wien2k [19]. Se usó el potencial de intercambio y correlación de Perdew-BurkeErnzerhof 96 [20] en la aproximación GGA. El método de solución de las ecuaciones de Kohn-Sham [21] que se usa en este código es el método SP-FP-LAPW y en él se usa como base para la expansión de la función de onda una base compuesta de ondas planas para 
la zona intersticial y para el interior de las esferas de muffin-tin se usa una combinación lineal de funciones radiales multiplicadas por armónicos esféricos. Para limitar el tamaño de la base, para las ondas planas se usó como corte de energía RMT x $\mathrm{K}_{\max }=7$ y para los armónicos esféricos se tomó como valor máximo del moméntum angular 10 . $\mathrm{G}_{\text {máx }}$, que es el máximo valor del vector del espacio recíproco para el desarrollo en serie del potencial fue 12. Los radios de muffin-tin (en u. a.) utilizados en este cálculos fueron, 2,50 para $\mathrm{Bi}, 1,85$ para $\mathrm{R}$ y para el $\mathrm{O} 1,61$. Se tomó una malla de 120 puntos en la parte irreducible de la primera zona de Brillouin. La convergencia de energía fue de 0,0001 Ry.

\section{Resultados}

En la figura 1 podemos ver la estructura de los compuestos de estudio. Las posiciones de los átomos son $\mathrm{Bi}(0,0,0) ; \mathrm{R}(1 / 2,1 / 2,1 / 2)$ y O $(0,1 / 2,1 / 2),(1 / 2,0,1 / 2)(1 / 2,1 / 2,0)$ y el grupo de espacio usado fue el \#221 (Pm-3m). Se observa que el $\mathrm{R}$ se encuentra en el centro de un octaedro determinado por 6 oxígenos.

Figura 1. Estructura de la perovskita $\mathrm{BiRO}_{3}$

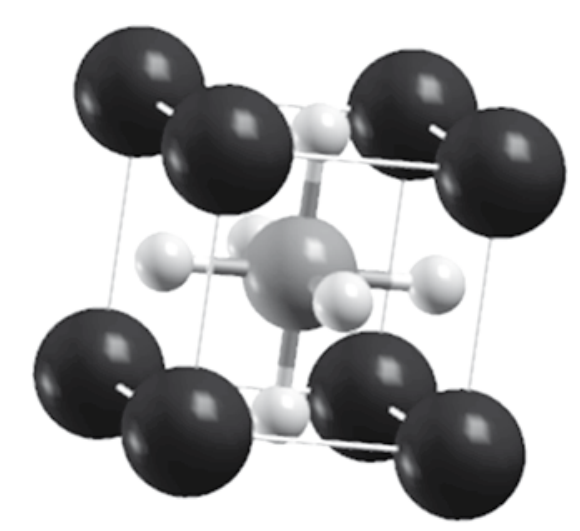

En la tabla 1 se resumen los resultados ab-initio obtenidos en la aproximación GGA para el compuesto del $\mathrm{BiRO}_{3}$ a $\mathrm{T}=0 \mathrm{~K}$, los cuales fueron ajustados con la ecuación de estado de Murnaghan [22]. Se presenta los parámetros de red, volumen de equilibrio, modulo de volumen y energía total. Podemos observar que el volumen disminuye a medida que aumenta el número atómico del elemento $\mathrm{R}$ en el compuesto $\mathrm{BiRO}_{3}$.

También se observa que la energía de equilibrio del compuesto $\mathrm{BiNiO}_{3}$, es más estable que los otros compuestos, una posible explicación a esta gran diferencia en energía, podrían ser el tipo de enlace que tienen los compuestos.

En la figura 2 reportamos las DOS del Compuesto $\mathrm{BiRO}_{3}$. Estos cálculos se realizaron para la constante de red correspondiente al volumen de equilibrio del estado base $(T=0$ K) de la estructura cúbica.

En ambas orientaciones del spin los materiales manifiestan un comportamiento metálico. Se observa que las DOS de los compuestos $\mathrm{BiFeO}_{3}$ y $\mathrm{BiCoO}_{3}$ son no simétricas, esto implica que debe existir un momento magnético total permanente cuyo valor es de $2,97 \mu_{\mathrm{B}}$, para el $\mathrm{BiFeO}_{3}$ y para $\mathrm{BiCoO} 3$ de $1,45 \mu_{\mathrm{B}}$. se puede observar que los responsable del magnetismo en el $\mathrm{BiFeO}_{3}$ son los orbitales d del Fe (en particular d-t2g). De igual forma sucede en $\mathrm{BiCoO}_{3}$ los responsable son los orbitales d (en particular d-t2g). Para el caso del compuesto $\mathrm{BiNiO}_{3}$, se observa que las DOS son simétricas y carácter conductor del compuesto está definido principalmente por los electrones $\mathrm{d}$ del $\mathrm{Ni}$.

Tabla 1. Volumen de equilibrio, parámetro de red, modulo de volumen y energía total de la perovskita $\mathrm{BiRO}_{3}$

\begin{tabular}{|cccccc}
\hline BiRO $_{3}$ & Grupo Espacial & Vo (u.a. ${ }^{3}$ ) & Parámetro de Red (u.a) & Bo (GPa) & Energía Total (eV) \\
\hline BiFeO $_{3}$ & Pm-3m & 386.035 & 7.281 & 167.342 & -22.139 \\
BiCoO $_{3}$ & Pm-3m & 374.555 & 7.208 & 187.081 & -20.614 \\
$\mathrm{BiNiO}_{3}$ & Pm-3m & 371.926 & 7.192 & 154.4199 & -226.984 \\
\hline
\end{tabular}


Figura 2. Densidad de estado total y parcial del compuesto $\mathrm{BiFeO}_{3}, \mathrm{BiCoO}_{3}$ y $\mathrm{BiNiO}_{3}$
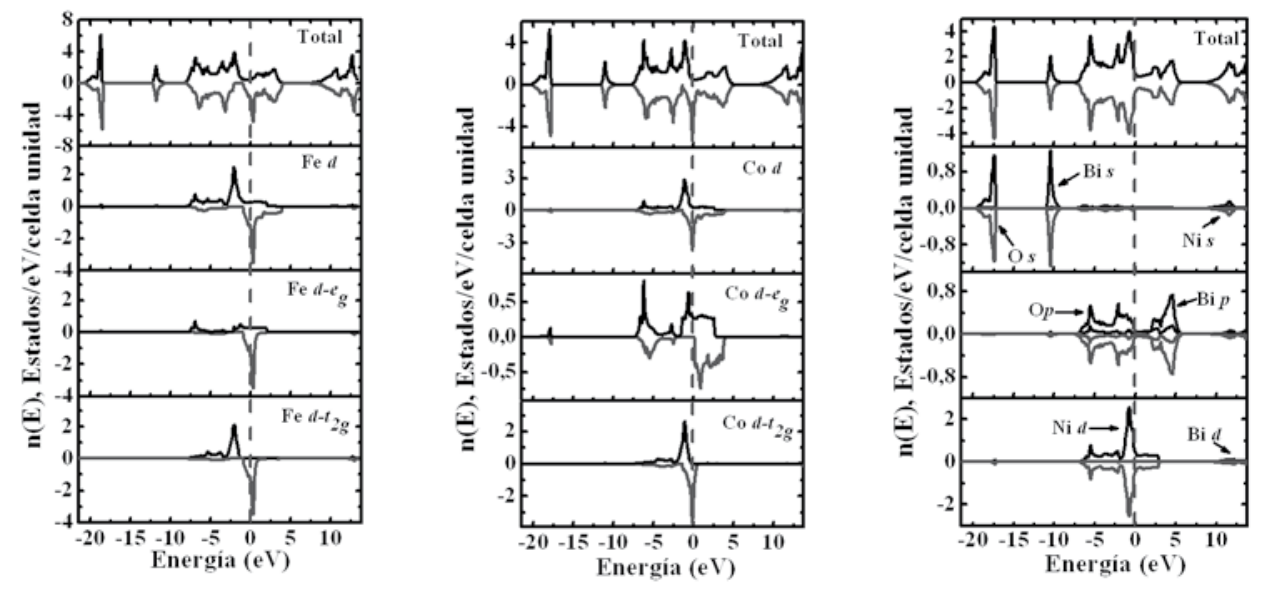

En la figura 3a hasta $3 \mathrm{c}$ se presenta la estructura de bandas para la polarización de spin up y para spin down a lo largo de algunas direcciones de alta simetría de la primera zona de Brillouin; en esta figura se observa la energía de los electrones en función del vector de onda $k$, tomado a lo largo de las direcciones $\mathrm{R}-\Delta-\Gamma-\Delta-\mathrm{X}-\mathrm{Z}-\mathrm{M}-\Sigma-\Gamma$. La estructura de bandas se calcula para la configuración en equilibrio; el cero de la energía se ha elegido en el nivel de Fermi. Se observa que las bandas de los compuestos $\mathrm{BiFeO}_{3}$ y $\mathrm{BiCoO}_{3}$ son diferentes para cada polarización de spin. Esto corrobora que debe existir un momento magnético permanente, mientras las del $\mathrm{BiNiO}_{3}$ son iguales. Lo cual fue examinado en las DOS. Además, se observa que el comportamiento del compuesto $\mathrm{BiRO}_{3}$, en la estructura de banda es muy similar. Por ejemplo, se observa una subanda interna de valencia que está ubicada en el rango entre los $-20 \mathrm{eV}$ y -18 eV. La principal contribución a esta subanda es debida a los electrones $s$ del O. En la parte superior de la banda de valencia, es decir la región comprendida entre $-12 \mathrm{eV}$ y $-9 \mathrm{eV}$ se observa una subanda muy definida perteneciente a los orbitales $s$ del Bi. Entre $-8 \mathrm{eV}$ y el Nivel de Fermi se observa una alta densidad de estados cuyas contribuciones son debidos a los electrones $p$ del $\mathrm{O}, d$ del Fe, Co y Ni. Inmediatamente arriba del nivel de Fermi, se encuentran contribuciones de los electrones $\mathrm{p}$ del $\mathrm{Bi}$ y minoritarias de $p$ del $\mathrm{O}$. En la parte superior, en la región entre $8 \mathrm{eV}$ y $12 \mathrm{eV}$ de la banda de conducción, la contribución es debida principalmente a orbítales $s \mathrm{Ni}$ y del p del Bi.

Figura 3a. Estructura de bandas para el $\mathrm{BiFeO}_{3}$ con Spin arriba y abajo.
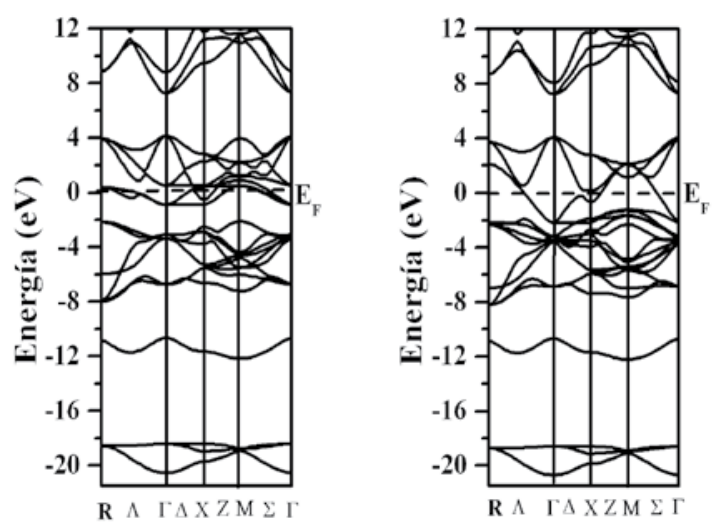

Figura 3b. Estructura de bandas para el $\mathrm{BiCoO}_{3}$ con Spin arriba y abajo.
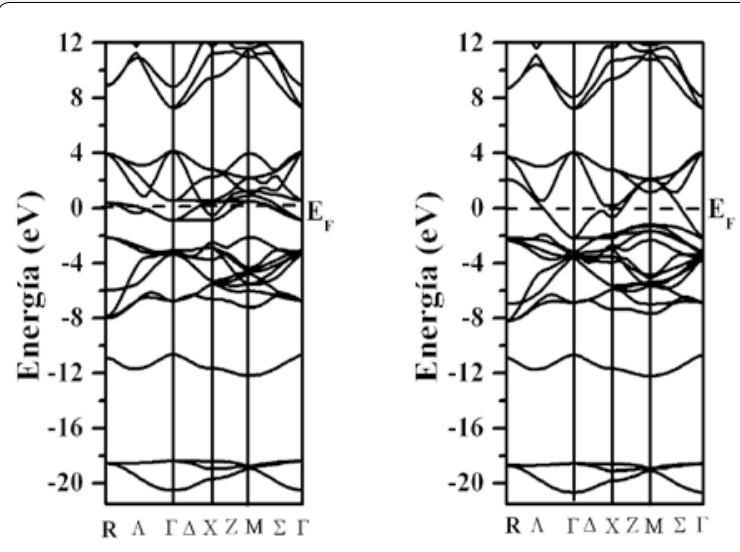
Figura 3c. Estructura de bandas para el $\mathrm{BiNiO}_{3}$ con Spin arriba y abajo.
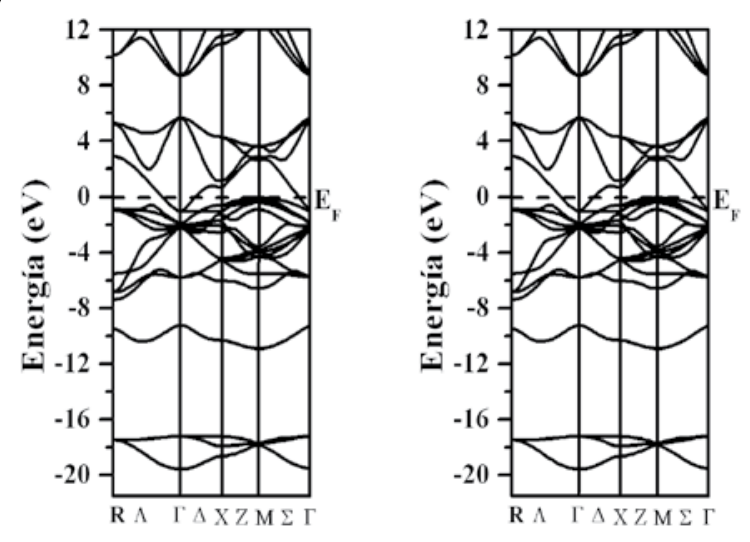

Se realizó un estudio de la $\Delta$ Energía vs Momento magnético fijo para el compuesto $\mathrm{BiNiO}_{3}$, y se verificó que la perovskita no presenta propiedades magnéticas. Como se

Figura 4. $\Delta$ Energía vs Momento Magnético fijo del compuesto $\mathrm{BiNiO}_{3}$

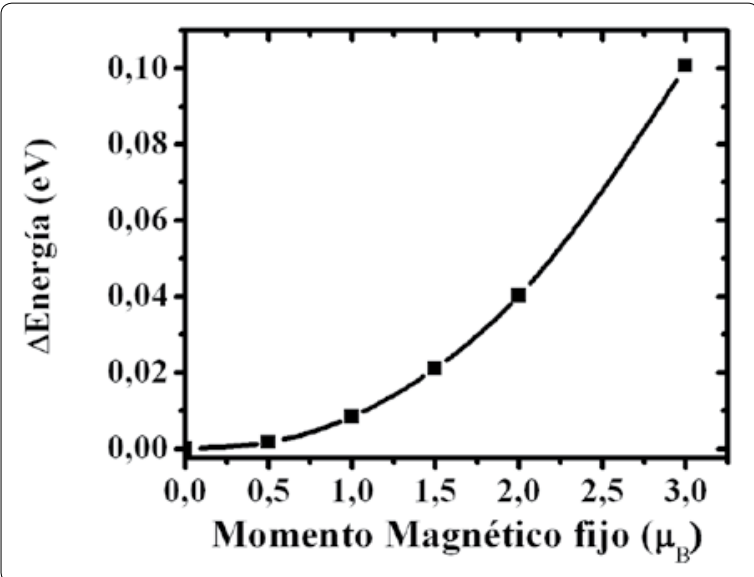

18 puede observar en la figura 4.

$\mathrm{BiNiO}_{3}$ no presenta un momento magnético. Se confirmaron que los tres materiales son metálicos y el comportamiento conductor se atribuye a los estados $d$ de cada mental de transición.

\section{Agradecimientos}

Este trabajo tuvo el apoyo parcial de Colciencias, proyecto No 1101-0617622, del Centro de Excelencia en Nuevos Materiales, contrato 043-2005 y de Fonciencias de la Universidad del Magdalena, proyecto No 002 -2007 .

\section{Referencias bibliográficas}

[1] M. Fiebig, T. Lottermoser, D. Fröhlich, A. V. Goltsev, and R. V. Pisarev, Nature (London) 419, 818 (2002).

[2] J. Wang, J. B. Neaton, H. Zheng, V. Nagarajan, S. B. Ogale, B. Liu, D. Viehland, V. Vaithyanathan, D. G. Schlom, U. V. Waghmare, et al., Science 299, 1719 (2003).

[3] T. Kimura, S. Kawamoto, I. Yamada, M. Azuma, M. Takano, and Y. Tokura, Phys. Rev. B 67, 180401(R) (2003).

[4] T. Kimura, T. Goto, H. Shintani, K. Ishizaka, T. Arima, and Y. Tokura, Nature (London) 426, 55 (2003).

[5] P. Baettig, N.A. Spaldin, Appl. Phys. Lett. 86, 12505 (2005).

Hemos analizado las propiedades estructurales, electrónicas y magnéticas del compuesto $\mathrm{BiRO}_{3}$ mediante DFT. Encontramos que a $\mathrm{T}=0 \mathrm{~K}$ los compuestos $\mathrm{BiFeO}_{3}$ y $\mathrm{BiCoO}_{3}$ tienen un momento magnético total permanente y los responsable de este momento magnético son los orbitales $d$ del Fe y Co respectivamente, mientras que el
[6] V. R. Palkar and S. K. Malik. Solid State Communications.134, 783 (2005).

[7] Alexei A. Belik, Hitoshi Yusa et al., Chem. Mater., 2009, 21 (14), pp 3400 3405. 
[8] Redfern, S. A. T.; Walsh, J. N.; Clark, S. M.; Catalan, G.; Scott J. F. 2009, arXiv:0901.3748v2.

[9] Y. Ya. Tomashpoískii, Y. N. Venevtsev, K. P. Burdina, and Y. N. Venevtsev, Kristallografiya, 13, 987 (1968).

[10] M. Q. Cai, G. W. Yanga and Y. L. Cao, W. H. Yu, L. L. Wang, and Y. G. Wang, Applied Phydics Letters 90, 242911 12007.

[11] Alexei A. Belik, Satoshi Likubo et al., Chem. Mater., 2006, 18 (3), pp 798 803.

[12] K. Oka, M. Azuma, Au. W. Chen et al., J.A. C. Soc 132 (27) 9438-9443 (2010).

[13] Meng-Qiu Cai, Ji-Cheng Liu et al. the Journal of Chemical Physics 126, 154708 (1-6) (2007).

[14] Feng Hong-Jian and Liu Fa-Min, Chinese Physics B Vol 18 No 4, 1574 1577 (2009).

[15] Feng Hong-Jian and Liu Fa-Min, Chin. Phys. Lett. Vol. 25, No. 2, 671-674 (2008)

[16] H. Wang et al. Solid state Comunication 149 (2009) 641-644.

[17] Masaki Takata, Makoto Sakata and Kenichi Kato, J. Mater. Chem.12, 3733 . 3737 (2002)

[18] P. Hohenberg, and W. Kohn, Phys. Rev. 136 (1964) 864

[19] P. Blaha, K. Schwarz, G.K.H. Madsen, D. Kvasnicka y J. Luitz, WIEN2k_08, A Full Potential Linearized Augmented Plane Wave Package for Calculating Crystal Properties. Karlheinz Schwarz, Techn. Universität Wien, Austria, 2001.
[20] PERDEW J. P (1996). Generalized Gradient Aproximation Made Simple, Phys. Rev. Letters 77, p. 3865.

[21] Kohn W. and Sham L.J. 1965 Phys. Rev. 140, A1133

[22] F. D. Murnaghan, Proc. Natl. Acad. Sci., USA. 30 (1944) 244. 